\title{
FRAMEWORK PARA AVALIAÇÃO DE USABILIDADE DE SISTEMAS BASEADO NO MÉTODO DE ANÁLISE HIERÁRQUICA DO PROCESSO
}

\section{FRAMEWORK FOR THE EVALUATION OF SYSTEMS USABILITY BASED ON THE METHOD ANALYTIC HIERARCHY PROCESS}

\author{
Cristiane Ellwanger, M.Sc. (1) \\ Cristina Paludo Santos, M.Sc. (2) \\ Joel Levandowski, Bel. (3) \\ (1) Universidade Regional Integrada do Alto Uruguai e das Missões \\ e-mail: cristianeellwanger@gmail.com \\ (2) Universidade Regional Integrada do Alto Uruguai e das Missões \\ e-mail:paludo@san.uri.br \\ (3) Universidade Regional Integrada do Alto Uruguai e das Missões \\ e-mail: jlevn@san.uri.br
}

Palavras-chave: Usabilidade, Análise Hierárquica do Processo, Heurística

\begin{abstract}
O presente artigo tem por intuito demonstrar a aplicabilidade do método AHP (Analytic Hierarchy Process) como auxílio ao processo de avaliação de usabilidade de sistema. Para tanto apresenta um framework capaz de subsidiar especialistas na realização destas avaliações, fazendo uso de heurísticas e atributos de usabilidade
\end{abstract}

\section{Keywords: Usability, Analytic Hierarchy Process, Heuristic}

This article demonstrates the applicability of AHP (Analytic Hierarchy Process) to improve the process of usability evaluation systems. Thereby presents a framework able to support specialists in implementation these evaluations, making use of heuristics and usability attributes

\section{Introdução}

A usabilidade de sistemas tem sido abordada em pesquisas de diversos autores e salientam que as interfaces a eles relacionadas devem ser desenvolvidas com vistas a proporcionar aos usuários não somente atingir seus objetivos, mas também aliar facilidade de aprendizagem e a satisfação destes em sua utilização. Entretanto, verifica-se a necessidade de apresentação de um método de avaliação de usabilidade que agregue em si métodos que sem voltem a tomada de decisão, visto que estes auxiliam a identificação de problemas a serem resolvidos prioritariamente. No intuito de suprir essa lacuna, o presente artigo 


\section{$16^{\circ}$ \\ ERGODESIGN USIHC CINAHPA}

propõe um Framework para avaliação da usabilidade, capaz de direcionar os aspectos relevantes que devem ser observados no que se refere à usabilidade de sistemas. Tal framework tem por base a Análise Hierárquica do Processo (AHP) e alia os estudos consagrados de Nielsen (NIELSEN, 1990; NIELSEN, 1994; NIELSEN, 2007) ao método de apoio à decisão.

Como contribuição o framework propicia não só no auxílio na identificação de problemas, mas também em determinar, de forma quantitativa, o quanto tais problemas afetam os usuários. Cabe destacar que este trabalho não se detém somente na apresentação do método, mas também na demonstração de sua utilização para posterior aplicação do mesmo em diferentes contextos, auxiliando profissionais de usabilidade em suas avaliações.

\section{O Framework FAU-AHP}

Levando-se em consideração as vantagens em se representar problemas complexos de forma hierárquica, o entendimento de que avaliações de usabilidade não são consideradas problemas triviais e a inegável importância do design para a qualidade dos sistemas desenvolvidos, é proposto um framework para avaliação da usabilidade de sistemas (FAU-AHP), tendo por base a AHP, conforme os pressupostos acima referenciados.

A categorização de problemas em problemas menores envolve a estruturação dos mesmos em níveis e a determinação de prioridades para a resolução de tais problemas. Tendo em vista que os atributos de usabilidade foram e permanecem sendo as bases sobre as quais foram criadas as heurísticas de usabilidade, propostas por Nielsen (1994, 2003), optou-se pela categorização das mesmas nos atributos a elas correspondentes, na determinação das prioridades a serem observadas com relação a atributos e heurísticas e no mapeamento de ações para se contemplar tais heurísticas, o que permite uma melhor elucidação dos problemas que permeiam o desenvolvimento de sistemas em torno da usabilidade.

Salienta-se, entretanto que tais categorizações e $16^{\circ}$ Ergodesign - Congresso Internacional de Ergonomia e Usabilidade de Interfaces Humano Tecnológica: Produto, Informações Ambientes Construídos e Transporte

$16^{\circ}$ USIHC - Congresso Internacional de Ergonomia e Usabilidade de Interfaces Humano Computador

CINAHPA | 2017 - Congresso Internacional de Ambientes Hipermídia para Aprendizagem.

priorizações foram embasadas nos estudos de Rocha; Baranauskas (2003) e Nielsen (1990, 1994, 2007), levando em consideração o contexto a ser aplicado. Assim, o Quadro 1, ilustra como os atributos de usabilidades (AU), as heurísticas (H) e as ações que visam contemplá-las (A), podem ser categorizadas.

\begin{tabular}{|l|}
\hline 1. AU- Facilidade de Aprendizagem \\
\hline 1.1 H - Prevenção de erros (PE) \\
1.1.1 A- Mensagens Claras - MC \\
1.2 H - Consistência e Padrões (CP) \\
1.2.1 A- Consistência e padronização de objetos e informações \\
(PA) \\
1.3 H - Relembrança ao invés de memorização (RM) \\
1.3.1 A - Reconhecimento de Links (LI) \\
1.4 H - Compatibilidade do sistema com o mundo real (CR) \\
1.4.1 A - Associações entre objetos, conceitos e informações - \\
IC. \\
\hline 2. AU- Facilidade de Relembrar (FR) \\
\hline 2.1 H - Compatibilidade do sistema com o mundo real (CR) \\
2.1.1 A- Associação de objetos reais com virtuais (AR) \\
2.2 H - Relembrança ao invés de memorização (RM) \\
2.2.1 A - Mudança nas cores de links após acesso (ML) \\
\hline 3. AU- Eficiência de Uso (EU) \\
\hline 3.1 H - Flexibilidade e eficiência de uso (FEU) \\
3.1.2 A- Atalhos (AT) \\
3.2 H - Controle e Liberdade do Usuário (CLU) \\
3.2.1 A- Controle sobre ações (CA) \\
3.3 H - Ajuda e documentação (AD) \\
3.3.1 A- Mapa do Site (MS) \\
3.3.2 A- Sistema de Busca (SB) \\
3.3.3 A- Informações de Contato (IC) \\
\hline 4. AU- Erros \\
\hline 4.1 H - Prevenção de erros (PE) \\
4.1.1 A- Orientação correta para entrada de dados (OD) \\
4.2 H - Ajudar os usuários a reconhecer, diagnosticar e corrigir \\
erros (AE) \\
4.2.1 A - Mensagens de erros compreensíveis (MC) \\
\hline 5. AU- Satisfação Subjetiva (SS) \\
\hline 5.1 H - Consistência e padrões (CP) \\
5.1.1 A - Vocabulário simples (VS); \\
5.1.2 A - Padronização das informações, das cores, ícones, \\
cabeçalhos e rodapés (PR); \\
5.2 H - Estética e design minimalista (EM) \\
5.2.1 A - Vocabulário simples (VS); \\
5.2.2 A - Padronização das informações, das cores, ícones, \\
cabeçalhos e rodapés (PR); \\
5.3 H - Visibilidade de status do sistema (VS) \\
5.3.1 A - Tempo de Carga (TP) \\
5.3.2 A - Navegabilidade (NG); \\
\hline
\end{tabular}

Quadro 1: Categorização de Heurísticas e atributos de usabilidade.

Adotou-se esta estruturação devido aos pressupostos iniciais em torno da usabilidade de sistemas, referenciada na literatura, se direcionar para atributos de usabilidade enquanto que evolução em torno de conceitualizações e de estudos consagrados voltados à interação humano computador, usabilidade e ergonomia têm gerado
Realização:

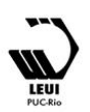




\section{$16^{\circ}$ \\ ERGODESIGN USIHC CINAHPA}

novos e muitos conceitos que, muitas vezes são similares e que confundem os projetistas e desenvolvedores no momento de sua utilização.

Conforme demonstra o quadro acima, as heurísticas de usabilidade foram integradas aos atributos de usabilidade a elas pertinentes ao mesmo tempo em que foram identificadas ações passíveis de serem executadas no momento em que são realizadas avalições de usabilidade no/durante o processo de desenvolvimento de sistemas.

Na medida em que se procede à categorização, acima exposta, compreende-se melhor as particularidades e/ou similaridades inerentes a atributos e heurísticas e as ações que possibilitam contemplá-los ou não no/durante o processo de desenvolvimento de sistemas.

Para a definição de pesos dos critérios de segundo nível (Facilidade de Aprendizagem, Facilidade de Relembrar, Eficiência de Uso, Erros e Satisfação Subjetiva) atribuiu-se os valores da escala de Saaty. Salienta-se que tais valores podem ser atribuídos de acordo com a percepção e nível de conhecimento pertinente a cada avaliador não somente com relação à usabilidade em si, mas também com relação ao entendimento do contexto de aplicação em que estas avaliações são realizadas.

A atribuição dos pesos dá origem a matrizes de comparação entre critérios. Após a definição das matrizes comparativas dos critérios para todos os subníveis da estrutura, procede-se à normalização dos pesos atribuídos. Para isso cada valor dos atributos mostrados nas colunas da matriz de julgamento é dividido pela sua respectiva soma na coluna. Normalizar os valores de cada coluna envolve o somatório de todos os seus elementos de forma que a soma dos mesmos corresponda ao valor 1 (resolução completa do problema).

Realizada a normalização de cada atributo pode-se através do mesmo obter seu peso individual (priorização de critérios).

\section{Estruturação do Framework Proposto}

Para a validação do framework o mesmo foi $16^{\circ}$ Ergodesign - Congresso Internacional de Ergonomia e Usabilidade de Interfaces Humano Tecnológica: Produto, Informações Ambientes Construídos e Transporte

$16^{\circ}$ USIHC - Congresso Internacional de Ergonomia e Usabilidade de Interfaces Humano Computador

CINAHPA | 2017 - Congresso Internacional de Ambientes Hipermídia para Aprendizagem.

aplicado na avaliação de usabilidade de um site web acadêmico, contando com a colaboração de cinco (5) avaliadores (usuários) que identificaram problemas relacionados à usabilidade.

Inicialmente, é apresentada a AHP definida a partir da ponderação dos valores com a análise dos mesmos, bem como o grau de severidade atribuído ao último nível da estrutura, conforme o julgamento dos avaliadores. No intuito de verificar o quanto algumas questões de usabilidade eram significativas para os usuários do site e de posse do percentual de relevância de um atributo em relação ao outro, correspondentes a cada nível da estrutura AHP, foi elaborado um questionário contendo 19 questões fechadas, referenciadas na Figura 1 por $\mathrm{P} n$, sendo que as possibilidades de respostas para as perguntas do questionário apresentam as variáveis PI (problema identificado) e PNI (problema não identificado).

Para a elaboração do questionário, utilizou-se uma escala Likert de 5 pontos percentuais, variando de "muito baixo" (1) a "muito alto" (5), no intuito de se verificar o grau de severidade com que determinados problemas de usabilidade são percebidos pelos usuários. Os cinco profissionais (usuários) responsáveis pela avaliação provêm, das áreas da Computação e Sistemas de Informação, Pedagogia e Matemática, considerando-se os pressupostos de Nielsen com relação ao número de avaliadores, pois conforme $\mathrm{o}$ autor, à medida que $\mathrm{o}$ número de avaliadores aumenta há uma tendência bastante alta de que problemas já identificados sejam mencionados novamente (NIELSEN, 2000). Além disso, a avaliação foi realizada por por profissionais pertencentes a diferentes áreas do conhecimento, uma vez que a usabilidade de sistemas é uma área multidisciplinar (ROCHA e BARANAUSKAS, 2003), (PREECE; ROGERS, 2005).

A Figura 4 demonstra a ponderação de valores, determinada a partir das matrizes demonstradas anteriormente dá origem a AHP, no qual um problema complexo (usabilidade de sistemas) pode ser desmembrado em problemas menores (atributos, heurísticas e ações) no intuito de se prover usabilidade aos sistemas.
Realização:

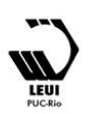




\section{$16^{\circ}$ \\ ERGODESIGN \\ USIHC CINAHPA}

$16^{\circ}$ Ergodesign - Congresso Internacional de Ergonomia e Usabilidade de Interfaces Humano Tecnológica: Produto, Informações Ambientes Construídos e Transporte

$16^{\circ}$ USIHC - Congresso Internacional de Ergonomia e Usabilidade de Interfaces Humano Computador

CINAHPA | 2017 - Congresso Internacional de Ambientes Hipermídia para Aprendizagem.

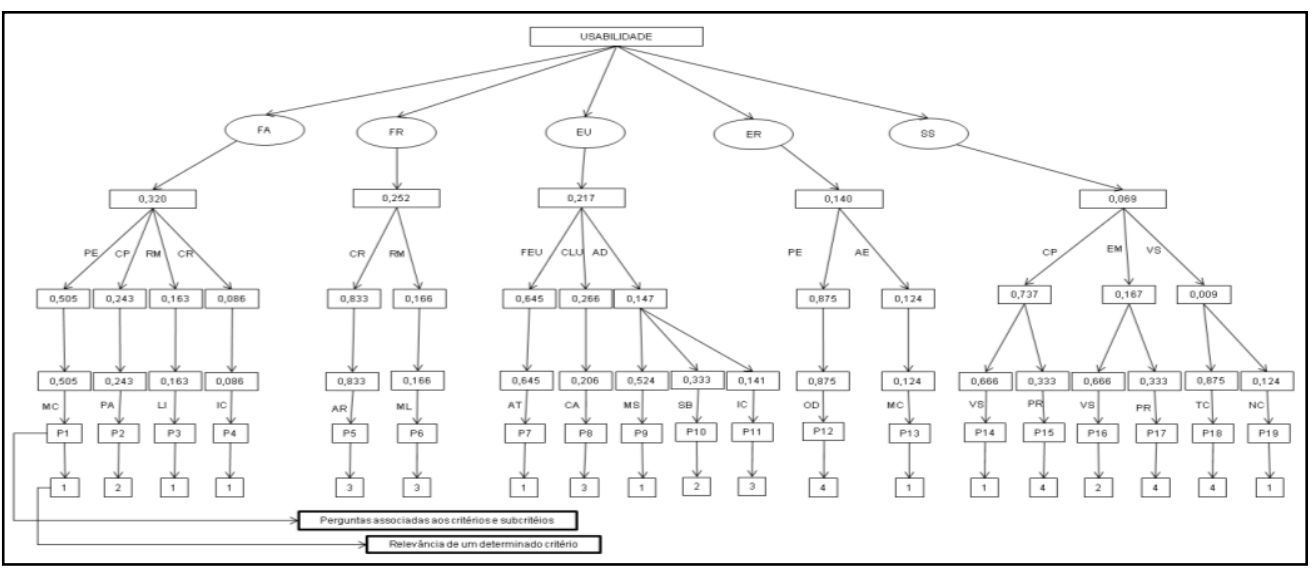

Figura 1: Pesos obtidos para o problema de usabilidade

\section{Resultados Obtidos}

A partir dos cálculos realizados e de posse dos valores correspondentes a cada nível da AHP, pode-se verificar através dos gráficos apresentados abaixo a relevância de um atributo em relação ao outro.

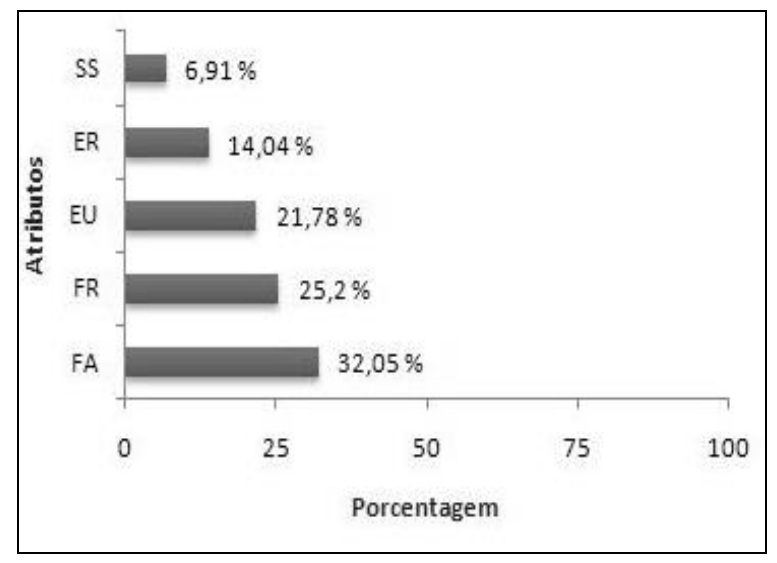

Figura 2: Gráfico de prioridade (segundo nível)

No gráfico da Figura 2, os atributos referentes ao segundo nível da estrutura AHP apresentam os seguintes percentuais: $32,05 \%$ para o atributo correspondente a facilidade de aprendizagem (FA), $25,2 \%$ para o atributo correspondente a facilidade de relembrar (FR), 21,78\% para o atributo correspondente eficiência de uso, $14,04 \%$ para o atributo referente a erros (ER) e por fim, 6,91\% correspondente ao atributo satisfação subjetiva (SS).

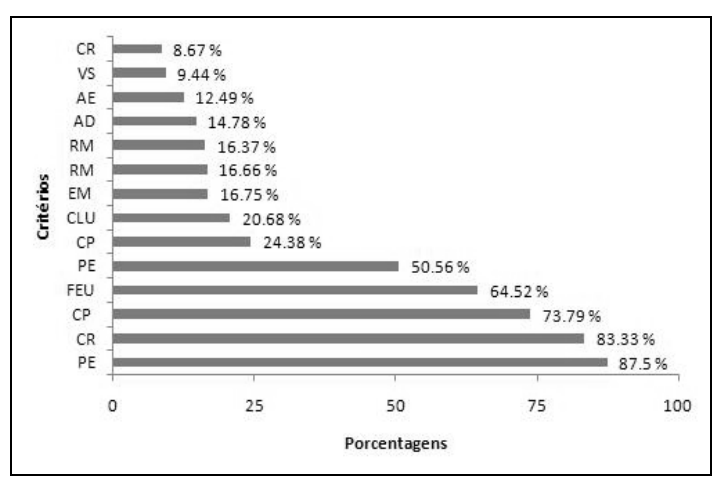

Figura 3: Gráfico de prioridade (terceiro nível)

Aos atributos do terceiro nível da estrutura, são apresentados no gráfico da Figura 3, no qual foram ponderados com os percentuais descritos conforme segue:

Facilidade de aprendizagem: 8,67\% para o atributo correspondente a compatibilidade do sistema com o mundo real $(\mathrm{CP}), 16,37 \%$ para o atributo correspondente a reconhecimento do sistema ao invés de memorização (RM), 24,38\% para o atributo correspondente consistência e padrões (CP), 50,56\% para o atributo correspondente a prevenção de erros (PE).

Facilidade de relembrar: 16,66\% para o atributo correspondente a compatibilidade do sistema com o mundo real (CR), 83,33\% para o atributo correspondente a reconhecimento ao invés de memorização (RM). 
$16^{\circ}$ Ergodesign - Congresso Internacional de Ergonomia e Usabilidade de Interfaces Humano Tecnológica: Produto, Informações Ambientes Construídos e Transporte

$16^{\circ}$ USIHC - Congresso Internacional de Ergonomia e Usabilidade de Interfaces Humano Computador

CINAHPA | 2017 - Congresso Internacional de Ambientes Hipermídia para Aprendizagem.
Eficiência de uso: $14,78 \%$ para o atributo correspondente a ajuda e documentação (AD), $20,68 \%$ para o atributo correspondente a controle e liberdade aos usuários (CLU), 64,52\% para o atributo correspondente a flexibilidade e eficiência de uso (FEU).

Erros: $12,49 \%$ para o atributo correspondente a ajudar os usuários a reconhecer, diagnosticar e corrigir erros (AE), 87,50\% para o atributo correspondente a prevenção de erros (PE).

Satisfação subjetiva: $9,44 \%$ para o atributo correspondente a visibilidade do status do sistema (VS), $16,75 \%$ para o atributo correspondente ao atributo estética e design minimalista (EM) e $73,79 \%$ para o atributo correspondente a consistência e padrão $(\mathrm{CP})$.

A partir da análise desses dados, pode-se verificar a significativa relevância dos atributos FEU, CP,

$\mathrm{CR}$ e $\mathrm{PE}$, os quais correspondem respectivamente à facilidade de uso, consistência e padrões, compatibilidade do sistema com o mundo real e prevenção de erros, apresentando percentuais acima de $50 \%$.

Estes percentuais, obtidos quantitativamente, permitem aos avaliadores demonstrar os motivos pelos quais determinados atributos merecem ser considerados prioritariamente para a resolução de problemas de usabilidade, justificando as decisões tomadas pelos mesmos.

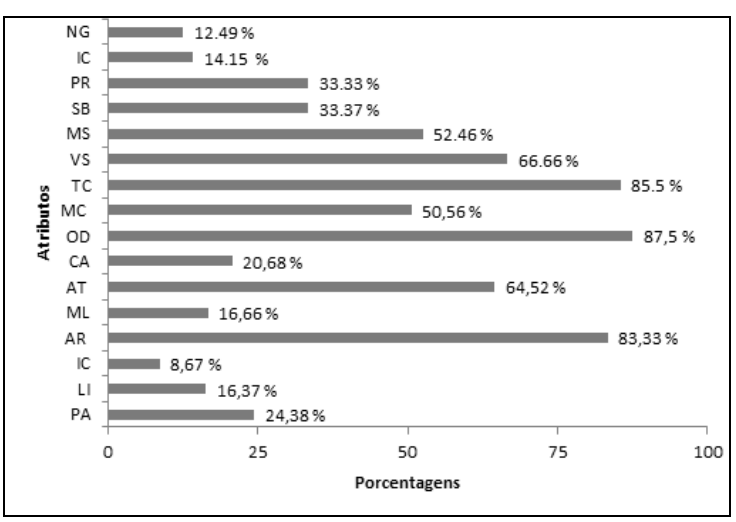

Figura 4: Gráfico de prioridade (quarto nível).

Conforme demonstrado no gráfico da Figura 4, os atributos referentes ao quarto nível da estrutura
AHP apresentam os seguintes percentuais: para os subcritérios Mensagens Claras (MC), padronização (PA), link (LI), ícones (IC), prevenção de erros (PE) e ajudar os usuários a reconhecer, diagnosticar e corrigir erros (AE) pertencentes conforme a sua ordem aos critérios prevenção de erros (PE), consistência e padrões (CP), reconhecimento ao invés de memorização (RM) e compatibilidade do sistema com o mundo real (CR) e erros (ER), o decisor não subdividiu os seus atributos, ou seja, os mesmos não foram comparados com nenhum outro atributo no mesmo nível da estrutura e no mesmo subcritério, sendo assim receberam os percentuais do nível acima ao qual pertenciam. Assim suas relevâncias correspondem a $50,56 \%, 024,38 \%, 16,37 \%$ e $8,67 \%$, respectivamente.

Aos critérios mudança de cores nos links (ML) e associação de um objeto real com virtual (AR), os quais pertencem aos subcritérios compatibilidade do sistema com o mundo real (CR) e reconhecimento ao invés de memorização (RM), atribuiu-se $83,33 \%$ e $16,66 \%$ de relevância devido aos mesmos motivos apresentados anteriormente.

Para os subcritérios atalhos (AT) e controle sobre as ações (CA), pertencentes aos critérios flexibilidade e eficiência de uso (FEU) e controle e liberdade aos usuários (CLU), atribui respectivamente $64,52 \%, 20,68 \%$. Já aos subcritérios Mapa do site (MS), Sistema de busca (SB), Informações para contato (IC) referente aos critérios ajuda e documentação (AD), atribuiu-se respectivamente $52,46 \%, 33,37 \%$ e $14,15 \%$ aos seus atributos.

Por fim, foram considerados para análise em níveis os subcritérios correspondentes ao vocabulário simples (VS) e padronização de informações, cores, ícones, cabeçalhos e rodapés (PR), pertencentes tanto para o critério consistência e padrões $(\mathrm{CP})$ quanto ao critério denominado Estética e Design Minimalista (EM), sendo atribuídos aos mesmos os percentuais de $66,66 \%$ e $33,33 \%$, respectivamente. Para os subcritérios tempo de carga (TC) e navegabilidade (NG) referente aos critérios visibilidade do status do sistema (VS) obtiveram-se, respectivamente, a 


\section{$16^{\circ}$ \\ ERGODESIGN USIHC CINAHPA}

estes atributos os seguintes valores $85,50 \% \mathrm{e}$ $12,49 \%$

Após a análise dos resultados advindos da aplicação do questionário e conjuntamente com as ponderações realizadas para com as mesmas, verificou-se que os problemas que devem ser corrigidos prioritariamente referem-se ao formato e a apresentação com que os dados e informações são disponibilizados no site, a marcação de links após a visita do usuário a uma determinada página, a padronização do layout do site, a apresentação de um mapa do site para que o usuário facilmente se localizar dentro do mesmo e a implantação de um sistema de busca para que o usuário encontre as informações de seu interesse de forma eficiente.

\section{Conclusões e Trabalhos Futuros}

Ao utilizar a Estrutura Analítica do Processo (AHP) como base, o framework proposto permite uma maior clareza na definição de um objetivo a ser alcançado (usabilidade de sistemas web), tendo em vista que a seleção da melhor opção pode ser bastante complexa, principalmente quando se tem um grande número de alternativas. Além disso, esta estrutura auxilia decisores em dois processos de suma importância:

a) no entendimento de preferências entre atributos, bem como se optou por uma determinada escolha em detrimento de outra, e

b) na documentação do processo relacionada a essa escolha, permitindo fornecer justificativas em torno de das alternativas escolhidas.

Embora o framework proposto retrate rigor metodológico, sua aplicação não exige grandes recursos tecnológicos, o que torna viável tais avaliações, havendo somente a necessidade de haver uma boa compreensão sobre usabilidade e dos pressupostos relacionados ao método AHP. Deste modo prevê-se que o processo de tomada de decisão retorne resultados satisfatórios, sendo determinados os ajustes e/ou alterações a serem realizados de forma prioritária.

Para trabalhos futuros sugere-se a aplicação do $16^{\circ}$ Ergodesign - Congresso Internacional de Ergonomia e Usabilidade de Interfaces Humano Tecnológica: Produto, Informações Ambientes Construídos e Transporte

$16^{\circ}$ USIHC - Congresso Internacional de Ergonomia e Usabilidade de Interfaces Humano Computador

CINAHPA | 2017 - Congresso Internacional de Ambientes Hipermídia para Aprendizagem.

método em diferentes contextos no intuito de se comprovar a viabilidade de sua aplicação em situações distintas. Além disso, sugere-se também o desenvolvimento de um ambiente computacional capaz agregar heurísticas e atributos de usabilidade com as características específicas do método AHP, o qual se detém na atribuição e ponderação de valores de acordo com o seu grau de relevância.

\section{Referências Bibliográficas}

NIELSEN, J., MOLICH, R. Heuristic evaluation of user interfaces. In: ACM Conference on Human Factors in Computing Systems. p. 249-256. Seattle, Washington, United States, 1990.

NIELSEN, J. Heuristic Evaluation: Usability Inspection Methods. John Wiley, New York, 1994

NIELSEN, J. Usabilidade na Web. Rio de Janeiro: Campus, 2007.

PREECE, J.; ROGERS, Y.; SHARP, H. Design de Interação: Além da interação homem-computador. Porto Alegre: Bookman, 2005.

ROCHA, H.V; BARANAUSKAS, M. C. C. Design e avaliação de interfaces humanocomputador. Campinas, SP: NIED/UNICAMP, 2003.

SAATY, T. L. How to make a decision: The Analytic Hierarchy Process. European Journal of Operational Research, v. 48, p. 9-26, 1990.
Realização:

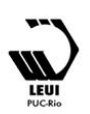

